\title{
ESTADO DE CONOCIMIENTO DE LOS PECES DULCEACUICOLAS DE CHILE
}

\section{CURRENT STATE OF KNOWLEDGE OF FRESHWATER FISHES OF CHILE}

\author{
Evelyn Habit ${ }^{1}$, Brian Dyer ${ }^{2} \&$ Irma Vila $^{3}$ \\ ${ }^{1}$ Unidad de Sistemas Acuáticos, Centro de Ciencias Ambientales EULA-Chile, Universidad de Concepción, \\ Casilla 160-C, Concepción, Chile. ehabit@udec.cl \\ ${ }^{2}$ Escuela de Recursos Naturales, Universidad del Mar, Amunátegui 1838, Recreo, \\ Viña del Mar, Chile. bdyer@udelmar.cl \\ ${ }^{3}$ Laboratorio de Limnología, Depto. Ciencias Ecológicas, Facultad de Ciencias, \\ Universidad de Chile, Santiago, Chile.limnolog@uchile.cl
}

\begin{abstract}
RESUMEN
La ictiofauna nativa de los sistemas límnicos de Chile se compone de 11 familias, 17 géneros y alrededor de 44 especies, incluyendo dos lampreas. De éstas, $81 \%$ son endémicas de la provincia biogeográfica chilena y $40 \%$ se encuentran clasificadas en peligro de extinción. Los grupos más representados corresponden a los órdenes Siluriformes (11 especies), Osmeriformes (9 especies) y Atheriniformes (7 especies). También están representados en Chile los ciclóstomos Petromyzontiformes (2 especies), y los teleósteos Characiformes (4 especies), Cyprinodontiformes (6 especies), Perciformes (4 especies) y Mugilifromes (1). Latitudinalmente, la mayor riqueza de especies ocurre en la zona centro-sur de la provincia Chilena, en tanto que los extremos norte y sur son de baja riqueza específica. Dado su origen, porcentaje de endemismo y retención de caracteres primitivos, este conjunto ictiofaunístico es de alto valor biogeográfico y de conservación. Existen sin embargo importantes vacíos de conocimiento sobre su sistemática, distribución y biología.
\end{abstract}

Palabras claves: Peces, sistemas dulceacuícolas, Chile.

\section{ABSTRACT}

The Chilean native freshwater ichthyofauna is composed of 11 families, 17 genera and about 44 species, including two lampreys. The Chilean biogeographic province has $81 \%$ of the species endemic, $40 \%$ of which are classified as endangered. The most abundant groups are the siluriforms (11 species), osmeriforms ( 9 species) and atheriniforms ( 7 species). Other groups represented in Chile are the cyclostomous Petromyzontiformes (2 species), and the teleostean characiforms (4 species), cyprinodontiforms (6 species), perciforms (4 species) and mugiliforms (1). The South Central area of the Chilean Province has the highest species richness, whereas the northern and southern areas are poorer in species numbers. Due to its origin, percentage of endemism and primitive characters, the ichthyological fauna of Chile has a high biogeographic and conservation value, however, there are important knowledge gaps in the systematics, distribution and biology of these freshwater fishes.

KEYwords: Fishes, freshwater systems, Chile. 


\section{INTRODUCCION}

La particular geomorfología de Chile, con barreras geográficas conformadas por la Cordillera de Los Andes, el Océano Pacífico y el Desierto de Atacama, ha generado una composición ictiofaunística continental única, caracterizada por ser poco diversa, presentar pequeños tamaños corporales, retener caracteres primitivos, alto endemismo y estar adaptada a ríos de alta pendiente y caudal fluctuante (Campos et al. 1993a, Ruiz \& Berra 1994, Vila et al. 1999a, Dyer 2000a). Los orígenes de esta ictiofauna son diversos. Algunas especies presentan relaciones con especies de ambientes tropicales, como es el caso de los bagres siluriformes del género Trichomycterus (Arratia 1997, Arratia \& MenuMarque 1981) y los characiformes del género Cheirodon (Campos 1982, Malabarba 1994). Por otra parte, los cyprinodontinos del género Orestias estarían emparentados con cyprinodontiformes anatolianos y de centro América (Parenti 1984, Costa 1997), mientras que otras especies tienen sus parientes más cercanos en Australia-Nueva Zelandia, tales como los galáxidos Galaxias y Aplochiton, los percoídeos del género Percichthys y las lampreas del género Geotria. En el caso de los atherinópsinos o pejerreyes sudamericanos de los géneros Odontesthes y Basilichthys, éstos están más cercanamente emparentados con pejerreyes atherinópsinos marinos de Norteamérica (Dyer 2000a,b).

Este trabajo entrega una visión general de la fauna íctica dulceacuícola de Chile, con énfasis en los grupos mejor representados y aquellos de mayor singularidad.

DIVERSIDAD, ENDEMISMO, DISTRIBUCIÓN Y ESTADOS DE CONSERVACIÓN.

Actualmente la fauna íctica de Chile se compone de un total de 11 familias, 17 géneros y alrededor de 44 especies nativas de peces estrictamente límnicos y diadrómicos, incluyendo dos especies de lampreas (Tabla I). Tanto a nivel de familias como de géneros, el grupo más importante en número es Siluriformes, con un total de 11 especies distribuidas desde el extremo norte hasta la Patagónia . Este grupo contiene representantes altamente singulares en Chile, tales como el género monotípico y endémico de la Provincia Chilena, Bullockia, cuya espe- cie B. maldonadoi presenta una distribución restringida en la zona Centro- Sur, entre los ríos Itata y Cautín. La familia Diplomystidae, representada por tres especies, está considerada como la más primitiva del orden Siluriformes (Arratia 1987, de Pinna 1998). Las especies de Diplomystes son altamente sensibles a las alteraciones de su hábitat, constituido primordialmente por las zonas ritrales de los ríos andinos del Centro-Sur de Chile (Arratia 1983b, Vila et al. 1999a, Habit 2005), por lo que presentan problemas de conservación. La fecundidad reportada para $D$. nahuelbutaensis, sumada a la presencia de huevos de gran tamaño (Vila et al. 1996), sugieren que esta especie podría presentar cuidado parental, sin embargo, Lundberg et al. (2004) describieron larvas libres de $13 \mathrm{~mm}$ y sin adaptaciones morfológicas. Otro siluriforme de gran singularidad es Nematogenys inermis, el que es considerado como el miembro más primitivo de la superfamilia Loricarioidea (Arratia 1992, de Pinna 1998) y por ser el que alcanza mayor talla en el grupo ( $400 \mathrm{~mm}$ LT). Esta especie ha sido descrita como un típico habitante de los potamones fluviales (Arratia 1983, Campos et al. 1993b), sin embargo, Ruiz (1993) la reporta como habitante de las zonas ritrales y potamales de la cuenca del río Andalién. Al igual que $D$. nahuelbutaensis, $N$. inermis presenta huevos de gran tamaño (4-4,5 mm) los cuales maduran sincrónicamente, indicando un único desove por temporada (Huaquin et al. 2002). Finalmente, destaca en el orden Siluriformes, el género Trichomycterus, correspondiente al de mayor riqueza de especies, distribución y abundancia. Sobresalen en este género especies de endemismo marcado, tales como $T$. chungarensis y T. laucaensis en la provincia Titicaca de la zona norte del país, y T. chiltoni presente sólo en las cuencas de los ríos Bío-Bío y Andalién. En tanto, T. areolatus es una especie de amplia distribución y muy abundante en ambientes de sustrato de gravilla y bolones, que abarca desde Huasco por el norte hasta la Isla Grande de Chiloé por el sur (Arratia et al.1981, Dyer 2000a).

Los Osmeriformes se encuentran representados también por un número importante de especies (9) en Chile, aún cuando Galaxias alpinus y Aplochiton marinus son consideradas como especies dudosas (Dyer 2000a). En este orden, Galaxias maculatus destaca por su distribución gondwánica, presentando la más amplia distribución disjunta entre todos los peces de agua dulce (Berra et al. 1996). 
Esta especie habita tanto en Australia, particularmente en Tasmania y Nueva Zelandia, como en Sudamérica (McDowall 1971a,b, Azpelicueta et al. 1996, Berra 2003). En Chile presenta poblaciones tanto diadrómicas como lacustrinas (Campos 1973 b y 1974), en las cuales se observa diferencias de tamaño en cuanto a sus huevos, embriones-libres y larvas metamórficas, así como en sus épocas de reproducción (Cussac et al. 2004). Galaxias platei tiene amplia distribución en la Patagonia, presentando adaptaciones a un clima altamente riguroso, tales como hábitos bentónicos en aguas profundas, retina adaptada a ambientes oscuros (con aguas de alta carga de sólidos), presencia de una línea lateral cefálica, protección de las branquias contra la abrasión, tolerancia a bajas de oxígeno y baja tasa metabólica (Cussac et al. 2004). Recientemente se han colectado individuos de G. platei de hasta $680 \mathrm{~g}$ y $36,8 \mathrm{~cm}$ de longitud total en la Región de Aysén. Galaxias globiceps, por otra parte, se encuentra restringida a un pequeño tributario del río Maullín (McDowall 1971a, Berra \& Ruiz 1994) y es considerada una especie rara (Campos et al. 1998). Berra y Barbour (1998), en base al estudio de ejemplares juveniles, plantean la problemática si esta especie está ya extinta o no. Sin embargo, el rango de distribución de esta especie ha sido recientemente ampliado por Murillo \& Ruiz (2002), incluyendo las localidades de Los Alerces cerca de Puerto Montt y en el río Cipresal ubicado en la costa occidental de la Isla de Chiloé. Las especies de Brachygalaxias por su parte se diferencian de B. bullocki por presentar una amplia distribución en la zona centro-sur, entre los ríos Itata y Maullín, en tanto $B$. gothei está fuertemente restringida a pequeños sectores en las cercanías de Talca. La validez de esta última especie fue discutida por Dyer (2000a). Las especies de Aplochiton tienen un rango de distribución latitudinal al sur de los $42^{\circ} 20^{\prime}$ en las cercanías del océano (Cussac et al. 2004), a excepción de $A$. zebra en la cuenca del río Bío-Bío, donde fue descrita como habitante de los lagos andinos Icalma y Galletué (Campos et al. 1993a). En número de especies, le siguen a Siluriformes y Osmeriformes el orden Atheriniformes, representado en Chile por 7 especies. Dyer (2000b) entrega una revisión completa de este grupo en Chile, manteniendo sólo una especie como du- dosa (Odontesthes (Cauque) itatanum). Los órdenes Cyprinodontiformes, Characiformes y Perciformes presentan cada uno 5 o 4 especies en Chile. El primero, representado por el género Orestias endémico de la provincia Titicaca, resulta altamente singular por su distribución disjunta y restringida a distintos salares (Arratia 1982a, Parenti 1984, Vila \& Pinto 1986). Recientemente, Vila (en prensa) ha propuesto una nueva especie de Orestias para Chile, la cual se encuentra en la Laguna Piacota.

Los Characiformes, representados por el género Cheirodon, se distribuyen alopátricamente en un sentido latitudinal. Así, Ch. pisciculus es una especie característica de la parte baja del río Maipo (Duarte et al. 1971), Ch. galusdae en los ríos Itata, Andalién y Bío-Bío (Campos et al. 1993b, Ruiz 1993, Habit 1998a, Habit et al. 2003) y Ch. australe en los "ríos andinos del sur de Chile" (sensu Campos 1985: Imperial, Toltén, Valdivia, Bueno y Maullín). También Ch. kiliani, una especie "rara" (sensu Campos et al. 1998), se distribuye en sectores costeros desde el Lago Lanalhue hasta el río Valdivia (Dyer 2000a). Estos peces viven generalmente en grandes grupos asociados a la vegetación acuática, donde se alimentan de las comunidades de auwfuchs o comunidades epibénticas (Victoriano \& Habit, 1993, Vila et al. 1999a). La presencia de $C h$. pisciculus entre los ríos Huasco y Maipo debe ser confirmada debido a que sólo hay registros actuales de la especie introducida Ch. interruptus. Los Perciformes están representados en Chile por los géneros Percichthys y Percilia, este último, endémico de la provincia Chilena (Dyer 2000a). Percichthys trucha presenta una amplia distribución latitudinal, entre Valparaíso y Tierra del Fuego, en tanto que $P$. melanops abarca entre Valparaíso y Concepción (Arratia et al. 1981, Arratia 1982b, Campos \& Gavilán 1996), donde han sido descritas como especies simpátricas (Campos et al. 1993). En tanto, las dos especies de Percilia ( $P$. gillissi y P. irwini) sólo han sido descritas en simpatría en la cuenca del río Andalién por Ruiz (1993). En el río Bío-Bío sólo estaría $P$. irwini, mientras $P$. gillissi se describe entre el río Aconcagua y el lago Llanquihue (Zunino et al. 1999, Vila et al. 1999a, Dyer 2000a).

Sin embargo, análisis moleculares indican que $P$. 
gillissi no es monofilética y que la taxonomía y patrones filogeográficos dentro del género Percilia, son más complejos de lo que se pensaba (Ruzzante et al. en prensa).

Finalmente, los ordenes Petromyzontiformes y Mugiliformes se encuentran representados por dos y una especie, respectivamente. El grupo de las lampreas está representado en Chile por las especies Geotria australis y Mordacia lapicida, siendo esta última endémica de Chile (McDowall 1988, Neira 1984). Ambas especies tienen distribuciones discontinuas desde el río Aconcagua a Tierra del Fuego, son parásitas y anádromas (Neira 1984, Campos et al. 1993a). En los ríos andinos y de la Cordillera de la Costa de la zona centro-sur es común encontrar larvas amocetes y macroftalmias en las zonas de sustrato fino y fangoso. Los adultos son escasos y sólo se registran temporalmente cuando suben a los ríos a reproducirse. El orden Mugiliformes está representado por Mugil cephalus, especie catádroma facultativa con una gran capacidad osmorregulatoria, la cual puede remontar hasta $50 \mathrm{~km}$ aguas arriba en los río andinos, como en el Itata (Habit 1998a).

El estado de conservación de la fauna de peces continentales de Chile fue revisado por un grupo de especialistas en 1998 (Campos et al. 1998, Tabla I). De esta revisión se concluye que este grupo de vertebrados presenta importantes problemas de conservación, estando sólo dos especies clasificadas como fuera de peligro (Ch. australe y M. cephalus). Entre las principales amenazas para la fauna de peces referidas a la alteración de hábitat se encuentran la construcción de embalses para centrales hidroeléctricas, la extracción de agua para riego, el vertido de residuos líquidos industriales y aguas servidas, la extracción de áridos, la canalización o semicanalización de los cauces, sustitución de bosque nativo por plantaciones forestales y contaminación difusa por pesticidas. Ejemplo de los impactos de algunas de estas acciones sobre la fauna nativa de peces han sido descritos para el funcionamiento de canales de riego (Habit \& Parra 2001), construcción y operación de centrales hidroeléctricas de paso (Habit et al. 2002), efecto de efluentes domésticos (Habit et al. 2005) y efectos acumulativos inducidos por acciones humanas (Habit et al. 2006). Estos trabajos muestran la disminución local de poblaciones de peces nativos y la alta capacidad de colonización de especies bentónicas como T. areolatus. La magnitud e importancia de los efectos de tales acciones sobre la fauna de peces nativos parece estar altamente relacionada con el grado de intervención previa en los sistemas acuáticos, presentando ambientes de condiciones más prístinas, comunidades más resistentes y resilientes (Habit et al. en prensa).

En cuanto a las amenazas biológicas, la de mayor impacto es el efecto de especies de peces introducidas ( 22 en el país), para las que en la mayoría de los casos se desconoce su real efecto sobre la ictiofauna nativa. Una mención especial tiene la invasión masiva de Oncorhynchus y Salmo, cuyos efectos en los ecosistemas límnicos chilenos es desconocido (Dyer 2000a, Gajardo \& Laikre 2002, Habit \& Rosenberger 2004). La depredación de especies nativas por parte de salmónidos ha sido reportada por Arenas (1978), Zama \& Cardenas (1982), Ruiz et al. (1993) y Ruiz \& Berra (1994), sin embargo, la magnitud e importancia de esta interacción no ha sido evaluada en Chile. El posible efecto negativo de salmonídeos sobre la distribución y abundancia de peces nativos en la zona sur de Chile ha sido discutido por Vila et al. (1999b) y Soto et al. (2003). Para ríos de la Isla Grande de Tierra de Fuego, Vila et al. (1999b) describieron una distribución fuertemente fragmentada de $G$. maculatus, sólo restringida a sectores con represas de castores (Castor canadienses), sugiriendo un efecto combinado de la presencia de salmonídeos y castores. Por su parte Soto et al. (2003) reportan la total ausencia de peces nativos en ríos desde los $41^{\circ} \mathrm{S}$ hasta Tierra del Fuego. La presencia y dominancia de salmonídeos en esta área geográfica (principalmente $S$. trutta), sugiere un desplazamiento de la fauna nativa por parte de los introducidos. De igual forma, Parra et al. (2003), estudiando el efecto de los niveles de trofía en lagos costeros de Chile central, encontraron un incremento de la riqueza de especies en ambientes de mayor trofía, pero con una mayor dominancia de especies introducidas tolerantes (e.g. Cyprinus carpio y Odontesthes bonariensis). El incremento de C. carpio y desplazamiento de especies nativas como $P$. trucha, $P$. melanops y $O$. mauleanum ha sido también detectado en ambientes de pozones con influencia de efluentes de celulosa en el río Bío-Bío (Habit et al. 2006). Por su parte, en zonas de ambientes someros del mismo río, el efecto de efluentes industriales implica el reemplazo de especies nativas como 
P. irwini por la especie introducida y tolerante Gambusia holbrooki (Habit et al. 2006). La introducción de la rana africana Xenopus leavis ha impactado notoriamente poblaciones de pejerreyes en el sector Colliguay del río Maipo y estero Limache del río Aconcagua (Dyer, obs. pers.). El efecto de esta introducción en los ríos de la zona central debe ser cuantificado.

\section{VACIOS DE CONOCIMIENTO}

Los principales vacíos de conocimiento sobre la ictiofauna dulceacuícola en Chile son los siguientes: 1) Se requieren estudios filogenéticos de los taxa nativos y especialmente de galaxiinos y Aplochiton. Además se requiere resolver las relaciones filogenéticas entre las especies de Diplomystes, resolver la identificación de las especies del género Orestias y de los pejerreyes del subgénero Cauque, así como las relaciones entre las especies que los componen. 2) Aclarar vacíos taxonómicos (ver especies dudosas en Tabla I). 3) Se requiere de un muestreo acabado de peces en los ríos Mataquito y Toltén, en las cuencas del área Atacameña y lagos y lagunas de la provincia Titicaca, así como en los sistemas del extremo sur del país. 4) Se requiere generar conocimiento de la biología y ecología de las especies nativas. Existe escasa o nula información sobre épocas de reproducción, fecundidad, estrategias reproductivas, edad, capacidad locomotora, migraciones, nicho trófico, distribución espaciotemporal a micro, meso y macroescala, carga íctica de ríos y lagos. 5) Se requieren estudios cuantitativos que analicen el efecto de especies introducidas en las nativas y de las acciones antrópicas que están causando la disminución de poblaciones (ver párrafos anteriores). Esta información es de vital importancia para adoptar medidas de conservación apropiadas para cada especie y sistema acuático.
ESPECIALISTAS EN EL GRUPO Y COLECCIONES DE REFERENCIA

En la Tabla II se indican los especialistas nacionales y extranjeros que actualmente se encuentran desarrollando investigación en algún grupo de peces limínicos chilenos o temática relacionada. No podemos dejar de mencionar al Dr. Hugo Campos Cereceda (q.e.p.d.), quién fue uno de los precursores del estudio de peces límnicos en Chile, y quien generó un valioso cuerpo de conocimiento, tanto en el ámbito de la taxonomía y sistemática como en la biología y ecología de la ictiofauna chilena.

La Tabla III muestra las principales colecciones de referencia en Chile y el extranjero, muchas de las cuales están disponibles on-line. Sería de gran utilidad para el desarrollo de la ictiología continental en Chile digitalizar las colecciones existentes en el país y hacerlas disponibles a través de páginas Web institucionales o colectivas, como es el caso de los proyectos NEODAT II (www.neodat.org) y FISHNET (http://speciesanalyst.net/fishnet/partners.html).

\section{CONCLUSION}

Aun cuando la ictiofauna de sistemas límnicos chilenos es pobre en especies, presenta un alto valor sistemático, biogeográfico y de conservación. Atentan contra su conservación los vacíos de conocimiento sobre su distribución, abundancia, sistemática y biología, necesarios para implementar adecuadas medidas de mitigación y manejo frente a las amenazas que mantiene un alto número de especies en riesgo de extinción (40\%).

\section{AGRADECIMIENTOS}

Este trabajo fue parcialmente financiado por el Proyecto DIUC Patagonia 205.310.042-15P. 
Peces dulceacuícolas de Chile: HaBit, E. ET AL.

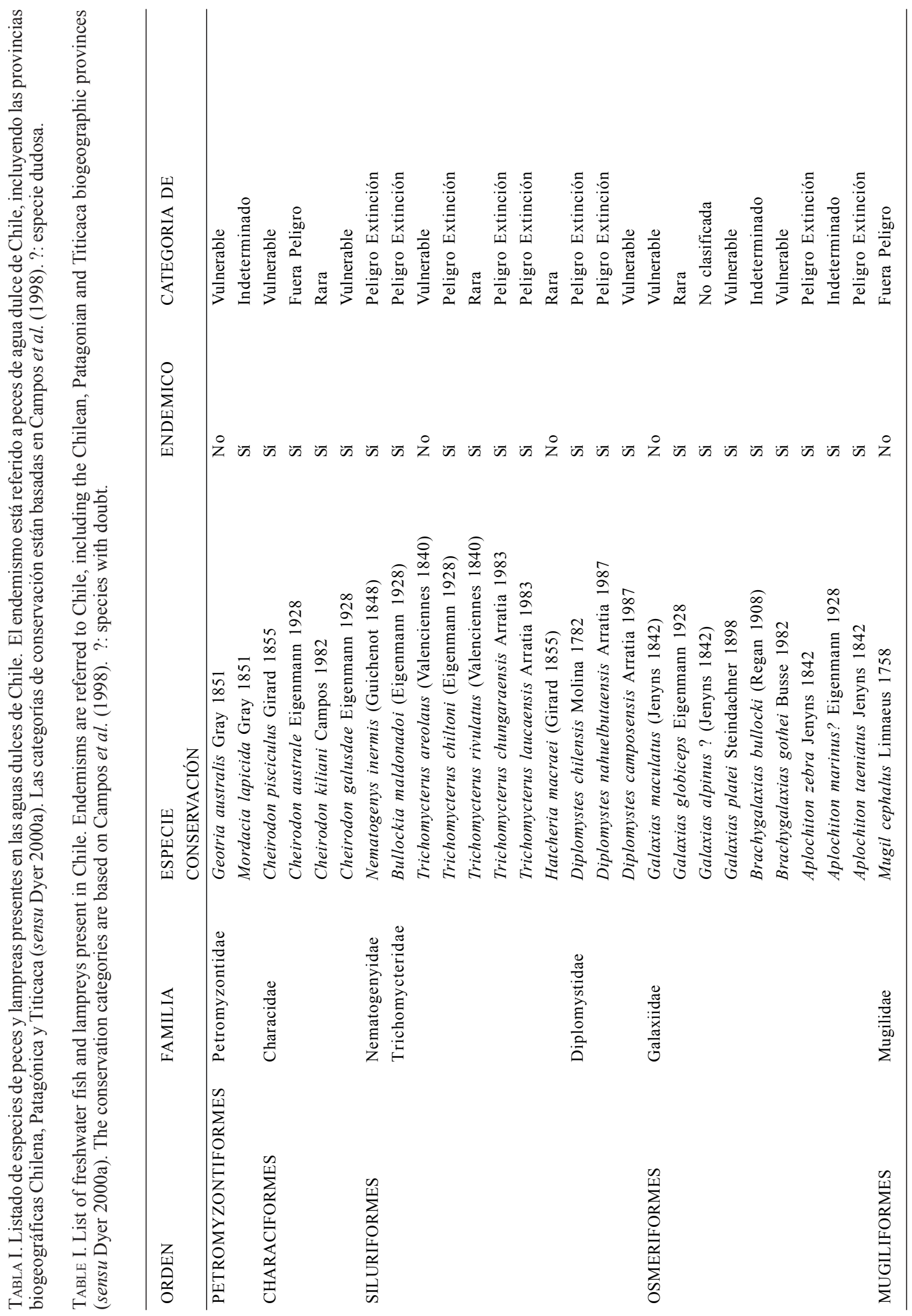


Gayana 70(1), 2006

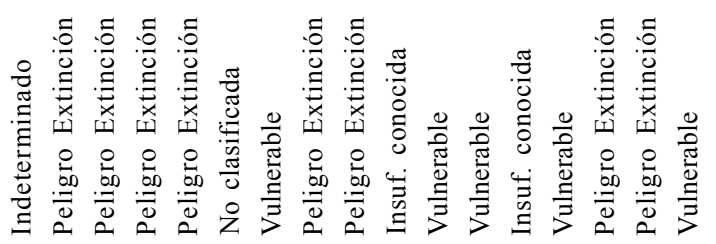

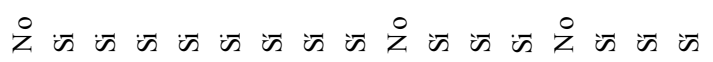

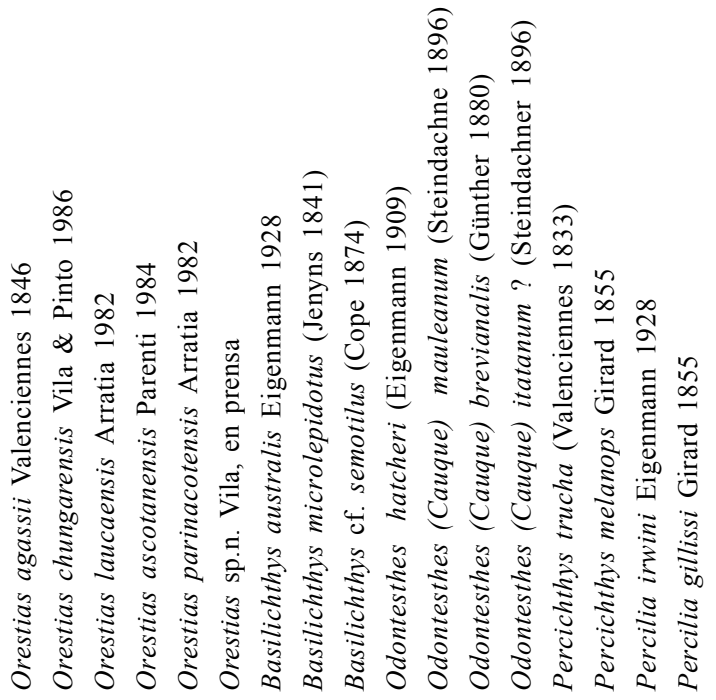

\begin{tabular}{|c|c|c|}
\hline 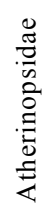 & 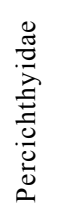 & 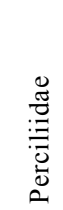 \\
\hline 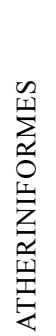 & 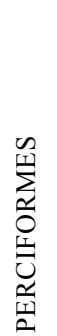 & \\
\hline
\end{tabular}


Peces dulceacuícolas de Chile: HaBIT, E. ET AL.

TABLA II. Especialistas en peces de agua dulce en Chile y en el extranjero.

TABLE II. Freshwater fish specialists in Chile and other countries.

\begin{tabular}{|c|c|c|}
\hline Nombre & Especialidad o Grupo de interés & Institución \\
\hline \multicolumn{3}{|l|}{ En Chile } \\
\hline Cuevas, César & Sistemática, Genética & Universidad Austral de Chile \\
\hline Dyer, Brian & Sistemática, Biogeografía, Filogenia & Universidad del Mar \\
\hline Habit, Evelyn & Ecología, Conservación & Universidad de Concepción \\
\hline Huaquín, Laura & Biología & Universidad de Chile \\
\hline Gajardo, Gonzalo & Genética & Universidad de Los Lagos \\
\hline Gavilán, Juan Francisco & Sistemática, Genética & Universidad de Concepción \\
\hline Quiroz, Sergio & $\begin{array}{l}\text { Ecología, Conservación } \\
\text { Valparaíso }\end{array}$ & Museo Historia Natural \\
\hline Ruiz, Víctor Hugo & Sistemática, Biología & Universidad de Concepción \\
\hline Soto, Doris & Ecología & Universidad Austral de Chile \\
\hline Victoriano, Pedro & Genética, Ecología & Universidad de Concepción \\
\hline Vila, Irma & Sistemática, Ecología, Conservación & Universidad de Chile \\
\hline Zunino, Sergio & Biología, Conservación & Universidad de Playa Ancha \\
\hline \multicolumn{3}{|l|}{ Fuera de Chile } \\
\hline Arratia, Gloria & Sistemática, Biogeografía, Filogenia & University of Kansas (KU) \\
\hline Berra, Tim & Biogeografía, Biología & Ohio State University \\
\hline Busse, Klaus & $\begin{array}{l}\text { Sistemática galáxidos } \\
\text { Koenig (ZFMK) }\end{array}$ & $\begin{array}{l}\text { Zoologisches Forschungsinstitut } \\
\text { und MuseumAlexander }\end{array}$ \\
\hline Cussac, Víctor & $\begin{array}{l}\text { Percichthyidae, Galaxiidae } \\
\text { Comahue, Bariloche }\end{array}$ & Universidad Nacional de \\
\hline Malabarba, Luiz & $\begin{array}{l}\text { Sistemática, Biogeografía characidos } \\
\text { Universidade Católica de Porto } \\
\text { (UFRGS) }\end{array}$ & $\begin{array}{l}\text { Museu Ciencias Pontificia } \\
\text { Alegre (MCP) y Universidade } \\
\text { Federal Rio Grande do Sul }\end{array}$ \\
\hline Mayden, Richard & Petromyzontidae & Saint Louis University \\
\hline Neira, Francisco & Petromyzontidae & Australia \\
\hline Ruzzante, Daniel & Percichthyidae, Galaxiidae & Dalhousie University \\
\hline
\end{tabular}


Gayana 70(1), 2006

TABLA III. Principales colecciones de peces de agua dulce en Chile y el exterior, y museos en línea.

TABLE III. Most important collections of Chilean freshwater fishes in Chile and in other countries, and online museums.

\begin{tabular}{|c|c|}
\hline Tipo de Colección & Institución \\
\hline \multicolumn{2}{|l|}{ Museo } \\
\hline MNHN & Museo Nacional de Historia Natural, Santiago (www.mnhn.cl). \\
\hline MZUC & Museo de Zoología, Universidad de Concepción, Concepción. \\
\hline MZUA & Museo de Zoología, Universidad Austral de Chile, Valdivia. \\
\hline \multicolumn{2}{|l|}{ Museo online } \\
\hline $\mathrm{AMNH}$ & $\begin{array}{l}\text { American Museum of Natural History (http://research.amnh.org/ichthyology/ } \\
\text { specimens.html) }\end{array}$ \\
\hline ANSP & $\begin{array}{l}\text { Academy of Natural Sciences, Philadelphia (http://data.acnatsci.org/ } \\
\text { biodiversity_databases/fish.php) }\end{array}$ \\
\hline CAS & $\begin{array}{l}\text { California Academy of Sciences, San Francisco (http://www.calacademy.org/ } \\
\text { research/ichthyology/collection/index.asp) }\end{array}$ \\
\hline $\mathrm{CU}$ & Cornell University (http://cuvc.bio.cornell.edu/cufish/index.html ) \\
\hline FMNH & $\begin{array}{l}\text { Field Museum of Natural History, Chicago (http://fm1.fieldmuseum.org/collections/ } \\
\text { search.cgi?dest=fish) }\end{array}$ \\
\hline KU & The University of Kansas, Natural History Museum (http://nhm.ku.edu/fishes/) \\
\hline MCZ & $\begin{array}{l}\text { Museum of Comparative Zoology, University of Harvard (http:// } \\
\text { collections.oeb.harvard.edu/Fish/FishSearch.htm) }\end{array}$ \\
\hline MNHN & $\begin{array}{l}\text { Museum National D'Histoire Naturelle, Paris (http://www.mnhn.fr/museum/foffice/ } \\
\text { science/science/ColEtBd/bdCollections/bdCollections/catalogue.xsp?SIGLE= } \\
\text { ichtyologie\&idx=7\&nav=liste) }\end{array}$ \\
\hline NRM & Swedish Museum of Natural History (http://artedi.nrm.se/nrmfish/search.php) \\
\hline SMNS & Staatliches Museum fur Naturkunde Stuttgart \\
\hline USNM & Smithsonian National Museum of Natural History (http://www.nmnh.si.edu/vert/ \\
\hline & fishes/fishcat/) http://www.nhm.org/research/fishes/ \\
\hline \multirow[t]{2}{*}{ UMMZ } & The University of Michigan Museum of Zoology (www.ummz.lsa.umich.edu) \\
\hline & ( http://141.211.243.52/UMMZ/) \\
\hline $\mathrm{ZMH}$ & Zoologische Museum Hamburg (http://www.rrz.uni-hamburg.de/cgi-bin/fischsearch) \\
\hline ZMB & Museum für Naturkunde der Humboldt Universität, Berlin \\
\hline \multicolumn{2}{|l|}{ Personales } \\
\hline UCV & Colección Laboratorio Morfología Funcional Animal, Cecilia Cancino, Valparaíso \\
\hline UMAR & Colección Universidad del Mar, Brian Dyer, Viña del Mar \\
\hline EULA & Colección Evelyn Habit, Universidad de Concepción, Concepción \\
\hline
\end{tabular}




\section{BIBLIOGRAFIA}

Arellano, M., L. Huaquín, A. Manríquez \& G. Arratia. 1980. Aspectos reproductivos en Trichomycterus aerolatus. Archivos de Biología y Medicina Experimentales 13: 43.

Arellano, M., L. Huaquín \& A. Manríquez. 1983. Aspectos comparativos de dos especies de Siluriformes Chilenos de agua dulce (Pisces, Trichomycteridae). Archivos de Biología y Medicina Experimentales 16: 136.

Arenas, J. 1978. Análisis de la alimentación de Salmo gairdneri Richardson en el lago Riñihue y río San Pedro, Chile. Medio Ambiente 3(2): 50-58.

Arratia, G. 1982a. Peces del altiplano de Chile. En: El Hombre y los ecosistemas de montaña (Eds. A. Veloso \& E. Bustos), pp. 93-133. Ediciones Oficina Regional de Ciencia y Tecnología de la UNESCO para América Latina y el Caribe. Montevideo, Uruguay.

Arratia, G. 1982b. A review of freshwater percoids from South America (Pisces, Osteichthyes, Perciformes, Percichthyidae, and Percillidae). Senckenberg Naturforsch Gesellsch 540: 1-52.

Arratia, G. 1983a. Trichomycterus chungaraensis n. sp. and Trichomycterus laucaensis n. sp. (Pisces, Siluriformes, Trichomycteridae) from the high Andean range. Studies on Neotropical Fauna and Environment 18: 65-87.

Arratia, G. 1983b. Preferencias de hábitat de peces siluriformes de aguas continentales de Chile (Fam. Diplomystidae y Trichomycteridae). Studies on Neotropical Fauna and Environment 18(4): 217-237

Arratia, G. 1987. Description of the primitive familiy Diplomystidae (Siluriformes, Teleostei, Pisces): morphology, taxonomy and phylogenetic implications. Bonner zoologische Monographien 24: 5-120.

Arratia, G. 1990. The South American Trichomycterinae (Teleostei: Siluriformes), a problematic group. En: Vertebrates in the tropics (Eds. G. Peters \& R. Hutterer), pp. 395-403. Museum Alexander Koenig, Bonn.

Arratia, G. 1992. Development and variation of the suspensorium of primitive catfishes (Teleostei: Ostariophysi) and their phylogenetic relationships. Bonner zoologische Monographien 32 :1-149.

Arratia, G. 1997. Brazilian and Austral fresh-water fish faunas of South America. A contrast. En: Tropical Biodiversity and Systematic (Ed. H. Ulrich), pp. 179-186. Proceedings of the international Symposium on Biodiversity and Sistematics in Tropical Ecosystem. Museum Alexander Koenig, Bonn.

Arratia, G., A. Chang, S. Menu-Marque \& G. Rojas. 1978. About Bullockia gen. nov,. Trichomycterus mendozensis n.sp. and revision of the family Trichomycteridae (Pisces, Siluriformes). Studies on Neotropical Fauna and Environment 13: 157-194.
Arratia, G. \& S. Menu-Marque. 1981. Revision of the freshwater catfishes of the genus Hatcheria (Siluriformes, Trichomycteridae) with commentaries on ecology and biogeography. Zoologische Anzeiger 207(1-2): 88-111.

Arratia, G., G. Rojas \& A. Chang. 1981. Géneros de peces de aguas continentales de Chile. Publicaciones ocasionales Museo Nacional de Historia Natural, Santiago de Chile 34: 3-108.

Azpelicueta, M., A. Almirón, A. López-Cazorla \& J. CAScIOTTA. 1996. Geographical distribution of Galaxias maculatus (Jenyns) (Pisces, Osmeriformes: Galaxiidae) in Patagonia. Biogeographica 72(4): 157-160.

Artigas J.N., E. CampuZano \& U. GonZÁlez. 1985. Contribución al conocimiento de la biología y hábitos alimentarios de Salmo gairdneri (Richardson, 1836) en Lago Laja (Chile). Gayana 49: 3 - 29.

Bahamonde, I., D. Soto \& I. Vila. 1979. Hábitos alimentarios de las especies de Atherinidae del embalse Rapel. Medio Ambiente (Valdivia, Chile) 4(1): 3-18

Berra, T. 2003. Family Galaxiidae (Galaxiids). En: Check list of the freshwaters fishes of South and Central America (Eds. R. Reis, S. Kullander \& C. Ferraris), pp. 503-506. Edipucrs, Porto Alegre, Brazil.

Berra, T. \& V.H. Ruiz. 1994. Rediscovery of Galaxias globiceps Eigenmann from southern Chile. Transactions of the American Fisheries Society 123: 595-600.

Berra, T., R. Feltes \& V.H. Ruiz. 1995. Brachygalaxias gothei from south-central Chile, a synonym of $B$. bullocki (Osteichthyes: Galaxiidae). Ichthyological Explorations of Freshwaters 6(3): 227-234.

Berra, T., L. Crowley, W. Ivantsoff \& P. Fuerst. 1996. Galaxias maculatus: an explanation of its biogeography. Marine and Freshwater Research 47: 845-849.

Berra, T. \& C. Barbour. 1998. Is the Chilean Galaxias globiceps (Teleostei: Galaxiidae) extant or extinct? Ichthyological Explorations of Freshwaters 9(3):273-278.

CAmpos, H. 1969. Reproducción de Aplochiton taeniatus Jenyns. Boletín del Museo Nacional de Historia Natural, Santiago de Chile 29: 207-222.

CAmpos, H. 1970. Introducción de especies exóticas y su relación con los peces de agua dulce de Chile. Noticiario Mensual del Museo Nacional de Historia Natural, Chile 14 (162) : 6-9.

CAmpos, H. 1972. Karyology of three Galaxiids fishes Galaxias maculatus, G. platei and Brachygalaxias bullocki. Copeia 2 :368-370.

CAmpos, H. 1973a. Lista de peces de aguas continentales de Chile. Noticiero Mensual del Museo Nacional Historia Natural, Santiago de Chile 17(198-199): 3-14.

Campos, H. 1973b. Migration of Galaxias maculatus (Jenyns) (Galaxiidae, Pisces) in Valdivia Estuary, Chile. Hydrobiologia 43: 301-312. 
Gayana 70(1), 2006

CAmpos, H. 1974. Population studies of Galaxias maculatus (Jenyns) (Osteichthyes: Galaxiidae) in Chile with reference to the number of vertebrae. Studies on Neotropical Fauna and Environment 9: 55-76

CAmpos, H. 1979. Avance en el estudio sistemático de la familia Galaxiidae (Osteichthyes: Salmoniformes). Archivos de Biología y Medicina Experimentales 12: 107-118.

CAmpos, H. 1982. Sistemática del género Cheirodon (Pisces: Characidae) en Chile con descripción de una nueva especie. Análisis de multivarianza. Studies on Neotropical Fauna and Environment 17: $129-162$

CAmpos, H. 1984a. Macrozoobentos y fauna íctica de las aguas limnéticas de Chiloé y Aysén continentales (Chile). Medio Ambiente 7(1): 52-64.

CAmpos, H. 1984b. Gondwana and neotropical galaxioid fish biogeography. Dr. W. Junk Publishers. The Hague: 113-125.

CAmpos, H. 1985. Distribution of the fishes in the Andean rivers in the South of Chile. Archives Hydrobiology 104 (2): 169 - 191.

Campos, H. \& C. Moreno. 1985. Asociaciones de peces en estuarios chilenos, Pacífico Sur Americano. En: Fish community ecology in estuaries and coastal lagoons: towards an ecosystem integration (En. L.A. Yánez-Arancibia), pp. 407-414. UNAM Press, México.

Campos, H. \& J. Gavilán. 1996. Diferenciación morfológica entre Percichthys trucha y Percichthys melanops (Perciformes: Percichthyiidae) entre $36^{\circ}$ y $41^{\circ}$ L.S. (Chile y Argentina) a través de análisis multivariado. Gayana Zoologúa 60(2): 99-120.

Campos, H., V.H. Ruiz, J.F. Gavilán \& F. Alay. 1993a. Peces del Río Bío-Bío. Serie Publicaciones de Divulgación EULA, Universidad de Concepción, Chile. 100 pp.

Campos, H., F. Alay, V.H. Ruíz \& J.F. Gavilán. 1993 b. Antecedentes biológicos de la fauna íctica presente en la hoya hidrográfica del río Bío-Bío. En: Seminario Limnología y Evaluación de Impacto Ambiental (Eds. O. Parra \& F. Faranda), pp. 7072. Ediciones Centro EULA-Chile, Universidad de Concepción, Chile.

Campos, H., J.F. Gavilán, V. Murillo \& P. Alarcón. 1996. Presencia de Cheirodon australe (Pisces: Characidae) en lago Tarahuin (Isla Grande de Chiloé, $42^{\circ} 40^{\prime} \mathrm{S}$, Chile) y su significado zoogeográfico. Medio Ambiente 13: 69-79.

Campos, H., G. Dazarola, B. Dyer, L. Fuentes, J.F. GAvilán, L. Huaquín, G. Martínez, R. Meléndez, G. Pequeño, F. Ponce, V.H. Ruiz, W. Siefeld, D. Soto, R. Vega \& I. Vila. 1998. Categorías de Conservación de peces nativos de aguas continentales de Chile. Boletín del Museo Nacional de Historia Natural, Santiago de Chile 47: 101-122.

Comte, S. \& I. VILA. 1986. Modalidad reproductiva de Basilichthys microlepidotus (Jenyns) en el río Choapa. (Pisces: Atherinidae). Anales del Museo
Historia Natural de Valparaíso 18: 85-94.

Comte, S. \& I. Vila. 1992. Spawning of Basilichthys microlepidotus (Jenyns). Journal of Fisheries Biology 41: 971-981.

CORFO. 1995. Potencialidad de las aguas interiores en la zona sur de la Provincia de Arauco (comunas de Cañete a Tirúa): Lagos Lanalhue y Lleulleu. Fondos Estudios e Investigaciones CORFO, Santiago de Chile. 320 pp.

Costa, W. 1997. Phylogeny and classification of the Cyprinodontidae revisited (Teleostei: Cyprininodontiformes): are Andean and Anatolian killifishes sister taxa? Journal of Comparative Biology 2(1): $1-17$.

Cussac, V., S. Ortubay, G. Inglesias, D. Milano, M. Lattuca, J.P. Barriga, M. Battini \& M. Gross. 2004. The distribution of South American galaxiid fishes: the role of biological traits and post-glacial history. Journal of Biogeography 31: 103-121.

Dazarola, G. 1972. Contribution a l' ètude de la faune ichtyologique de la region Valparaíso Aconcagua (Chili). Annals de Limnologie, Paris 8 (1): 87100.

De LA Hoz, E. 1994. Aspectos cinemáticos del mecanismo de mordida premaxilar en los géneros Cauque, Basilichthys, y Austromenidia (Teleostei, Atherinidae). Investigaciones Marinas, Valparaíso 22: 31-37.

De LA Hoz, E. 1995. El mecanismo de mordida premaxilar en Atherinopsinae (Teleostei: Atheriniformes): aspectos estructurales relacionados con la transmisión de fuerzas. Revista Chilena Historia Natural 68: 351-359.

De la Hoz, E. \& R. Aldunate. 1994. Variaciones del complejo tendinoso del fascículo A1 del músculo aductor mandibular y sus consecuencias en el mecanismo de mordida premaxilar en Cauque, Austromenidia, y Basilichthys (Teleostei, Atherinidae). Revista de Biología Marina, Valparaíso 29(2): 235-249.

De la Hoz, E., C. Cancino \& E. OJeda. 1994. Capacidades de modulación y plasticidad funcional de los mecanismos de captura de alimento en Atherinopsinae sudamericanos (Teleostei, Atherinidae). Investigaciones Marinas, Valparaíso 22: 45-65.

De la Hoz, E. \& E. Tosti-Croce. 1981. Osteología de Basilichthys australis Eigenmann (TeleosteiAtherinidae). Anales del Museo de Historia Natural de Valparaíso 14: 229-245.

De La Hoz, E. \& J. Vial. 1994. Diseño estructural bucal en pejerreyes sudamericanos (Atherinopsinae; Atherinidae): Modelo biocinemático de mecanismos de alimentación. Revista Chilena Historia Natural 67: 35-47.

De Pinna, M. 1998. Phylogenetic relationships of neotropical siluriformes (Teleostei: Ostariophysi): historical overview and synthesis of hypotheses. En: Phylogeny and Classification of Neotropical Fishes (Eds. L.R. Malabarba, R.E. Reis, R.P. Vari, 
Z.M. Lucena \& C.A. Lucena), pp. 279-330. Edipucrs, Porto Alegre, Brasil.

Duarte, W., R. Feito, C. Jara, C. Moreno \& A.E. Orellana. 1971. Ictiofauna del sistema hidrográfico del río Maipo. Boletín del Museo Nacional de Historia Natural, Chile 32: 227-268.

Dyer, B. 1997. Phylogenetic revision of Atherinopsinae (Teleostei, Atherinopsidae), with comments on the systematics of the South American freshwater genus Basilichthys Girard. Miscellaneous Publications, Museum of Zoology, University of Michigan 187: $1-64$.

DYER, B. 2000a. Systematic review and biogeography of the freshwater fishes of Chile. Estudios Oceanológicos, Chile 19: 77-98.

DYER, B. 2000b Revisión sistemática de los pejerreyes de Chile (Teleostei, Atheriniformes). Estudios Oceanológicos, Chile 19: 99-127.

DYer, B. 2003. Family Atherinopsidae. En: Check list of the freshwater fishes of South and Central America (Eds. R.E. Reis, S.O. Kullander \& C.J. Ferraris), pp. 515-525. Ediciones Edipucrs, Porto Alegre, Brasil.

Eigenmann, C. 1927. The freshwater fishes of Chile. Memories of the National Academy of Sciences 22: $1-63$.

Fowler, H. 1943. Fishes of Chile. Systematic Catalog. Revista Chilena de Historia Natural 54: 22-57.

Fowler, H. 1944. Fishes of Chile. Systematic Catalog. Revista Chilena de Historia Natural 56: 275-343.

Fowler, H. 1951. Analysis of the fishes of Chile. Revista Chilena de Historia Natural 63: 263-326.

GAJARDO, G.M. 1988. Genetic divergence and speciation in Basilichthys microlepidotus Jenyns, 1842 and $B$. australis Eigenmann, 1927 (Pisces, Atherinidae). Genetica 76: 121-126.

Gajardo, G.M. 1992. Karyotypes of Basilichthys microlepidotus and B. australis (Pisces: Atherinidae). Copeia 19: 256-258.

Gajardo, G. \& L. Laikre. 2002. Chilean aquaculture boom is based on exotic Salmon resources: a conservation paradox. Conservation Biology 17(4): 1173-1174.

HaBit, E. 1994. Contribución al conocimiento de la fauna íctica del río Itata. Boletín de la Sociedad de Biología de Concepción, Chile 65: 143 - 147.

HABIT, E. 1994. Ictiofauna en canales de riego de la cuenca del río Itata durante la época de otoño - invierno. Comunicaciones del Museo de Historia Natural de Concepción, Chile 8: 7- 12.

Habit, E. 1998a. Peces. En: Documento de síntesis estudio de línea de base para la evaluación de impacto ambiental del Complejo Forestal Industrial Itata (Eds. O. Parra \& E. Habit), pp. 79-86. Ediciones Centro EULA-Chile, Universidad de Concepción. 172 pp.

HaBit, E. 1998b. Análisis de la dieta de Percilia gillissi en ambientes de río y canales de riego (cuenca del río Itata, Chile). Theoria 7: 33-46.

HaBIT, E. 2005. Aspectos de la biología y hábitat de un pez endémico de Chile en peligro de extinción (Diplomystes nahuelbutaensis Arratia, 1987).
Interciencia 30: 8-11.

HABIT, E. \& O. PARRA. 2001. Impactos ambientales de los canales de riego sobre la fauna de peces. Ambiente y Desarrollo 17(3): 50 - 56.

Habit, E., P. Victoriano \& O. Parra. 2002. Translocación de peces nativos en la cuenca del río Laja (Región del Bío-Bío, Chile). Gayana 66: 181-190.

Habit E., P. Victoriano \& A. Rodriguez-Ruiz. 2003. Variaciones espacio-temporales del ensamble de peces de un sistema fluvial de bajo orden del centro sur de Chile. Revista Chilena de Historia Natural 76: 3-14.

Habit, E. \& A. Rosenberger. 2004. Introduced species in Chile's freshwaters-the need for research. Newsletter of the Introduced Fish Section American Fisheries Society 21(1):3-4.

Habit, E., O. Parra, C. Valdovinos. 2005. Ictiofauna de un sistema fluvial receptor de aguas servidas: respuestas a una nueva planta de tratamiento (río Quilque, Chile Central). Gayana Zoología 69: 94103.

HABIT, E. \& M. BeLK. 2005 Threatened fishes of the world: Percilia irwini Eigenmann, 1927 (Perciliidae). Environmental Biology of Fishes (en prensa).

Habit, E., P. Victoriano \& H. Campos. 2005. Ecología trófica y aspectos reproductivos de Trichomycterus areolatus (Pisces, Trichomycteridae) que colonizan ambientes lóticos artificiales. Revista Biología Tropical 52(4): 195 - 210.

Habit, E. \& P. Victoriano. 2005. Peces de agua dulce de la Cordillera de la Costa. En: Historia, Biodiversidad y Ecología de la Cordillera de la Costa de Chile (Eds. C. Smith-Ramírez, J. Armesto \& C. Valdovinos), pp. 392-406. Editorial Universitaria, Santiago, Chile.

Habit, E., C. Tuckfield \& O. Parra. 2006. Response of the fish community to human-induced changes in of the Bío-Bío river in Chile. Freshwater Biology, 51: 1-11.

Habit, E., M. Belk, O. Parra. 2006. Response of the riverine fish community to the construction and operation of a diversion hydropower plant in central Chile. Aquatic conservation: Marine and Freshwater ecosystems 16: 00-00.

Huaquín, L.G., D. Veliz \& G. Arratia. 2002. Estudio comparativo de ovarios y cubiertas ovocitarias en peces siluriformes de aguas continentales de Chile. Gayana Zoología 66(2): 269 - 274.

KLINCK, A. \& R. ECKMANN. 1985. Age and growth, feeding habits, and reproduction of Cauque mauleanum (Steindachner 1896) (Pisces: Atherinidae) in southern Chile. Studies Neotropical Fauna Environment 20(4): 239-249.

LundBerg, J., T. Berra \& J. Friel. 2004. First description of small juvenile of the primitive catfish Diplomystes (Siluriformes: Diplomystidae). Ichthyology Exploration Freshwaters 15: 71 - 82.

Malabarba, L.R. 1994. Sistemática e filogenia de Cheirodontinae (Ostariophysi: Characiformes: Characidae). Tesis Doutorado, Universidade de 
Sao Paulo, Brasil. 289 pp.

Manríquez, A., L. Huaquín, M. Arellano \& G. Arratia. 1988. Aspectos reproductivos de Trichomycterus areolatus Valenciennes, 1846 (Pisces: Teleostei: Siluriformes) en río Angostura, Chile. Studies on Neotropical Fauna and Environment 23: 89 102.

McDowall, R. 1971a. The galaxiid fishes of South America. Zoological Journal of the Linnean Society 50: 33-73.

Mcdowall, R. 1971b. Fishes of the family Aplochitonidae. Journal of the Royal Society of New Zealand 1(1): 31-52.

Mcdowall, R. 1976. The taxonomic status of the Galaxias populations in the Río Calle-Calle, Chile (Pisces: Galaxiidae). Comments on a proposal for specific recognition. Studies on Neotropical Fauna and Environment 11: 173-177.

Mcdowall, R. 1988. Diadromy in fishes. Migrations between freshweater and marine environments. Timber Press, Portland, Oregon. 308 pp.

Moreno, C., R. Urzua \& N. Bahamondes. 1977. Breading, sexual rate and fecundity of Basilichthys australis Eigenmann 1927, from Maipo River, Chile (Atherinidae, Pisces). Studies on Neotropical Fauna and Environment 12 : 217223.

Murillo, V. \& V.H. Ruiz. 2002. El puye Galaxias globiceps Eigenmann 1927 (Osteichthyes: Galaxiidae): ¿una nueva especie en peligro de extinción? Gayana Zoología 66(2): 191-197.

NeIRA, F. 1984. Biomorfología de las lampreas parásitas chilenas Geotria australis Gray, 1851 y Mordacia lapicida (Gray, 1851) (Petromyzontiforms). Gayana Zoología 48: 3-40.

Oliver, C. 1949. Catálogo de los peces fluviales de la provincia de Concepción. Boletín de la Sociedad de Biología de Concepción, Chile 24: 5160.

Olmos, V., P. Victoriano, E. Habit \& C. Valdovinos. 2003. Parásitos de peces nativos de la cuenca del río Laja (Chile central) y alcances sobre su ciclo de vida. Archivos de Medicina Veterinaria 35 (2): 195-203.

Palma, A., R. Figueroa, V.H. Ruiz, E. Araya \& P. Berrios. 2002. Composición de la dieta de Oncorhynchus mykiss (Walbaum 1792) (Pisces: Salmonidae) en un sistema fluvial de baja intervención antrópica: estero Nonguén, VIII Región, Chile. Gayana Zoología 66: 129-139.

PARDO, R. 2002. Diferenciación morfológica de poblaciones de Trichomycterus areolatus Valenciennes 1846 (Pises: Siluriformes: Trichomycteridae) de Chile. Gayana Zoología 66: 203-205.

Parenti, L. 1984. A taxonomic revision of the Andean killifish genus Orestias (Cyprinodontiformes, Cyprinodontidae). Bulletin of the American Museum of Natural History 178(2): 107-214.

Parra, O., C. Valdovinos, R. Urrutia, M. Cisternas, E. Habit, M. Mardones \& E. Ugarte. 2003. Ca- racterización y tendencias tróficas de cinco lagos costeros de Chile. Limnetica, España 22(1-2): 5183.

Pequeño, G. 1989. Peces de Chile. Lista sistemática revisada y comentada. Revista de Biología Marina, Valparaíso 24: 1-132.

Peredo, S. \& C. Sobarzo. 1994. Actividad gonádica estacional de Galaxias maculatus (Jenyns, 1842) en el río Cautín, IX Región, Chile. Boletín de la Sociedad de Biología de Concepción, Chile 65: 65-70.

Pinto, M. \& I. ViLA. 1987. Relaciones tróficas del género Orestias en el sistema hidrográfico Lauca. Anales del Museo de Historia Natural de Valparaíso 18: 77-84.

Prochelle, O. \& Campos H. 1985. The biology of the introduced carp Cyprinus carpio L., in the river Cayumapu, Valdivia, Chile. Studies on Neotropical Fauna and Environment 20(29): 6582.

Ruiz, V.H. 1993. Ictiofauna del río Andalién (Concepción, Chile). Gayana Zoología 57(2): 109-278.

Ruiz, V.H., M.T. López, H.I. Moyano \& M. Marchant. 1993. Ictiología del alto Biobío: Aspectos taxonómicos, alimentarios, reproductivos y ecológicos con una discusión sobre la hoya. Gayana Zoología 57: 77-88.

RuIz, V.H. 1996. Ictiofauna del río Laja (VIII Región, Chile): Una evaluación preliminar. Boletín de la Sociedad de Biología de Concepción 67: 15-21.

RuIz, V.H. \& T. BERRA. 1994. Fishes of the high Biobio river of south-central Chile with notes on diet and speculations on the origin of the ichthyofauna. Ichthyology Exploration Freshwaters 5: 5-18.

Ruzzante, D., S. Walde, V. Cussac, M. Dalebout, J. Seibert, S. Ortubay \& E. Habit. 2006. Phylogeography of the Percichthyidae (Pisces) in Patagonia: Roles of Orogeny, Glaciation, and Volcanism. Molecular Ecology (en prensa).

ScAsso, F. \& H. CAmpos. 1998. Oncorhynchus mykiss (Pisces, Salmonidae) populations in lakes of different trophic levels of the Biobío river basin, Chile. International Verein Limnologie 26: 2320-2323.

Scasso, F. \& H. Campos. 1999. Comparison of two populations of silverside (Odontesthes bonariensis) in Eutrophic lakes of Central Chile. Journal of Freshwater Ecology 14(1): 61-70.

Soto, D., I. Arismendi, E. Guzmán, J. GonZÁlez, C. JarA, S. Zelada, E. Neira \& A. Lara. 2003. Distribución, abundancia y potencial efecto de las especies salmonídeas sobre la fauna íctica nativa en el sur de Chile. Resumen XII Taller Nacional de Limnología, Concepción, Chile. :68.

Urzúa, R., C. Díaz, E. Karmy \& C. Moreno. 1977. Alimentación natural de Basilichthys australis en Tejas Verdes, Chile. Biología Pesquera, Chile. 9: 45-61.

Victoriano, P. \& E. Habit. 1993. Estudio de la dieta de Cheirodon galusdae (Pisces, Characidae) en un 
sistema léntico de la zona central de Chile. Libro Resúmenes VI Congr. Iberoamericano Conservación y Zoología de Vertebrados. Santa Cruz, Bolivia: 3 .

VILA, I. 2005. A new species of Killifish (Teleostei: Cyprinodontidae) from the southern high Andes, Chile. Copeia (en prensa).

Vila, I., \& D. Soto. 1981. Atherinidae (Pisces) of Rapel reservoir, Chile. International Verein Limnologie 21:1334-1338

Vila, I., D. Soto \& I. BAhamondes. 1981. Age and growth of Basilichthys australis (Eigenmann 1927) in Rapel reservoir, Chile. Studies on Neotropical Fauna and Environment 16: 9-22.

Vila, I. \& D. Soto. 1984. Odontesthes bonariensis "pejerrey argentino", una especie para cultivo extensivo. FAO Documento técnico 4: 224 - 228.

Vila, I. \& M. Pinto. 1986. A new species of killifish (pisces, Cyprinodontidae) from the Chilean Altiplano. Revista de Biología Tropical 19(3-4): 233-239.

Vila, I., M. Contreras \& L. Fuentes. 1996. Reproducción de Diplomystes nahuelbutaensis Arratia 1987 (Pises: Diplomystidae). Gayana Oceanología 4(2): 129-137.
Vila, I., L. Fuentes \& M. Contreras. 1999a. Peces Límnicos de Chile. Boletín del Museo Nacional de Historia Natural, Chile 48: 61-75.

VILA, I., L. Fuentes \& M. SAavedra. 1999b. Ictiofauna en los sistemas limínicos de la Isla Grande, Tierra del Fuego, Chile. Revista Chilena de Historia Natural 72: 273-284.

Welcomme, R.L. 1988. International introductions of inland aquatic species. FAO, Fisheries Technical Paper 294: 1-318.

ZAMA,A. \& E. CÁRDENAS. 1982. Seasonal occurrence of fishes collected in Ensenada Baja, Southern Chile, with notes of stomach contents, sex ratio and maturity. Introduction into Aysen Chile of Pacific Salmon. Documento Técnico Servicio Nacional de Pesca, Valparaíso $5: 1-16$.

Zunino, S.O., M. Baeza, S. Quiroz \& R. Rivera. 1999. Ampliación distribucional de la carmelita Percilia gillissi Girard, 1854 (Pisces: Perciliidae). Anales del Museo de Historia Natural de Valparaíso 24: 119120.

ZuÑIGA, L. \& P. EsCOBAR. 1993. Selectividad alimentaria de estados juveniles de Cauque mauleanum (Pisces, Atherinidae) en la Laguna de Quintero, Valparaíso, Chile. Acta Limnologica Brasiliensia 6: 157-162.

Fecha de recepción: 30.08 .05

Fecha de aceptación: 19.12.05 